\title{
ELASTIC-PLASTIC BOUNDARIES IN THE PROPAGATION OF PLANE AND CYLINDRICAL WAVES OF COMBINED STRESS*
}

\author{
BY
}

T. C. T. TING

University of Illinois at Chicago Circle

\begin{abstract}
A general study is given of plane and cylindrical wave propagation of combined stress in an elastic-plastic medium. The coefficients of the governing differential equations, when written in matrix notation, are symmetric matrices and can be divided into submatrices each of which has a special form. The relations between the stresses on both sides of an elastic-plastic boundary are derived. Also presented are the restrictions on the speed of an elastic-plastic boundary.
\end{abstract}

1. Introduction. The equations of motion for a continuum body are

$$
\partial \sigma_{i j} / \partial x_{i}=\rho \partial v_{i} / \partial t \quad(i, j=1,2,3)
$$

where $\sigma_{i j}$ is the stress, $v_{i}$ the velocity and $\rho$ is the mass density of the body, and summation is implied by repeated indices. The relation between the strain $\epsilon_{i i}$ and the velocity $v_{i}$ is

$$
\partial \epsilon_{i j} / \partial t=\frac{1}{2}\left(\partial v_{i} / \partial x_{i}+\partial v_{i} / \partial x_{i}\right),
$$

while the stress-strain relation for an elastic, isotropic work-hardening material is (see [1])

$$
\frac{\partial \epsilon_{i j}}{\partial t}=\frac{1+\nu}{E} \frac{\partial \sigma_{i j}}{\partial t}-\frac{\nu}{E} \delta_{i j} \frac{\partial \sigma_{k k}}{\partial t}+G(k) \frac{\partial f}{\partial \sigma_{i i}} \frac{\partial f}{\partial \sigma_{k l}} \frac{\partial \sigma_{k l}}{\partial t} ;
$$

$E$ is Young's modulus, $\nu$ is Poisson's ratio, $k$ is the yield stress and the yield condition can be written as

$$
f\left(\sigma_{i j}\right)=k^{2} .
$$

$G(k)$ in Eq. (3) is a given function of $k$ which characterizes the work-hardening property. Eqs. (1)-(4) give a complete description of wave propagation in three-dimensional elastic-plastic media.

In this paper, we will restrict our attention to special cases in which the governing equations depend on only one space variable. Plane wave propagation and cylindrical wave propagation are such special cases. The most general plane wave propagation is the one in which $v_{1}, v_{2}$, and $v_{3}$ are functions of $x_{1}$ and $t$ only. Using $x$ instead of $x_{1}$ for simplicity, $\sigma_{1}, \tau_{2}, \tau_{3}$ for $\sigma_{11}, \sigma_{21}, \sigma_{31}$ respectively, Eq. (1) gives

$$
\begin{aligned}
& \sigma_{1, x}=\rho v_{1, t}, \\
& \tau_{2, x}=\rho v_{2, t}, \\
& \tau_{3, x}=\rho v_{3, t},
\end{aligned}
$$

where the subscripts $x$ and $t$ denote partial differentiation with respect to these variables.

*Received December 15, 1968; revised version received February 3, 1969. 
If we use von Mises' yield condition

$$
f=\frac{1}{2} s_{i j} s_{i j}=k^{2}
$$

where

$$
s_{i j}=\sigma_{i j}-\frac{1}{3} \delta_{i j} \sigma_{k k},
$$

Eq. (3) gives, making use of Eq. (2) and the fact that $\epsilon_{22}=\epsilon_{33}=0$ leads to $\sigma_{22}=\sigma_{33}$ in the present case,

$$
\begin{aligned}
v_{1, x} & =\frac{1}{E} \sigma_{1, \iota}-\frac{2 \nu}{E} \sigma_{2, \iota}+s_{1} G Q, \\
0 & =-\frac{2 \nu}{E} \sigma_{1, t}+\frac{2(1-\nu)}{E} \sigma_{2, \imath}+2 s_{2} G Q, \\
v_{2, x} & =\tau_{2, \iota} / \mu+2 \tau_{2} G Q, \\
v_{3, x} & =\tau_{3, t} / \mu+2 \tau_{3} G Q,
\end{aligned}
$$

where $s_{1}, s_{2}$ and $\sigma_{2}$ stand for $s_{11}, s_{22}$, and $\sigma_{22}$ respectively, $\mu$ is the shear modulus and

$$
Q=s_{1} \sigma_{1, t}+2 s_{2} \sigma_{2, t}+2 \tau_{2} \tau_{2, t}+2 \tau_{3} \tau_{3, t} .
$$

Eqs. (5)-(11) give a complete description of a general plane wave propagation in an elastic-plastic medium.

When $v_{2} \equiv 0$, and hence $\tau_{2} \equiv 0$, Eqs. (5), (7), (8), (9), and (11) reduce to the case of pressure-shear wave propagation considered in [2], [3], [4]. If $v_{2}, v_{3}$ are the only nonzero velocity components, then $\tau_{2}$ and $\tau_{3}$ are the only nonzero stresses and Eqs. (6), (7), (10) and (11) reduce to the case of two-shear waves studied in [3], [5], [6]. The equations derived in [7] for combined longitudinal and torsional waves in a thin-walled tube may also be reduced from Eqs. (5), (6), (8) and (10) by letting $\sigma_{2} \equiv 0$.

If $v_{r}, v_{\theta}, v_{z}$ are the velocity components of a particle in cylindrical coordinates $(r, \theta, z)$, the most general cylindrical wave propagation is the one in which $v_{r}, v_{\theta}$ and $v_{z}$ are functions of $r$ and $t$ only. Then, the only nonzero strains are $\epsilon_{r}, \epsilon_{\theta}, \gamma_{\theta}, \gamma_{z}$ where $\gamma_{\theta}, \gamma_{z}$ stand for $\epsilon_{r \theta}, \epsilon_{r z}$ respectively. Consequently $\sigma_{r}, \sigma_{\theta}, \sigma_{z}, \tau_{\theta}, \tau_{z}$ are the nonzero stresses where $\tau_{\theta}=\sigma_{r \theta}, \tau_{z}=\sigma_{r z}$. Now, instead of writing the governing equations for cylindrical wave propagation in the form shown in Eqs. (5)-(11), we will use matrix notation and write the governing equations, Eqs. (1)-(3), in a matrix differential equation which can be applied to both plane and cylindrical wave propagation.

2. The matrix differential equation. For wave propagation which involves only one space variable $x$ or $r$, the equations of motion (1) can be written in matrix notation as

$$
\mathbf{M} \boldsymbol{\delta}_{x}+b_{1}=\rho v_{t}
$$

where $\boldsymbol{\delta}$ and $\mathbf{v}$ are column vectors whose elements are stress and velocity components respectively. $b_{1}$ is also a column vector whose elements are functions of stress $\boldsymbol{\delta}$ and space variable $x$ only. (In cylindrical waves, $x$ becomes $r$.) If $\boldsymbol{o}$ has $m$ elements and $\mathbf{v}$ has $n$ elements, then $\mathbf{M}$ is an $n \times m$ matrix whose elements are constants. The continuity condition Eq. (2) can be written as

$$
\varepsilon_{t}=\mathrm{Nv}_{x}+\mathrm{b}_{2}
$$


where $\varepsilon$ is a column vector with strains as its elements while $b_{2}$ is a column vector whose elements are functions of velocity $\mathbf{v}$ and space variable $x$ only. $\mathbf{N}$ is an $m \times n$ matrix whose elements are constants. Finally, the stress-strain relation, Eq. (3), can be written as

$$
\varepsilon_{t}=\mathbf{S} \boldsymbol{\sigma}_{\boldsymbol{t}}
$$

where $\mathbf{S}$ is an $m \times m$ square matrix. In the plastic region, $\mathbf{S}$ can be written more precisely as

$$
\mathbf{S}^{p}=\mathbf{S}^{e}+G(k)(\nabla f)(\nabla f)^{T} .
$$

$\mathbf{S}^{\circ}$ is also an $m \times m$ square matrix whose elements are functions of elastic constants only. Thus

$$
\varepsilon_{t}=\mathbf{S}^{e} \boldsymbol{\sigma}_{\boldsymbol{t}}
$$

gives the elastic stress-strain relation. $\nabla f$ is the gradient of $f(\boldsymbol{\sigma})$ with respect to the components of $\boldsymbol{\delta}$. Hence, by (4),

$$
(\nabla f)^{T} \boldsymbol{\sigma}_{t}=2 k k_{t} .
$$

Now, by eliminating $\varepsilon_{t}$ between Eqs. (14) and (15), we can write Eqs. (13)-(15) in one matrix equation

$$
\mathrm{Aw}_{t}+\mathrm{Bw}_{x}=\mathrm{b}
$$

where

$$
\begin{array}{ll}
\mathbf{A}=\left[\begin{array}{cc}
\rho \mathrm{I} & 0 \\
0 & \mathrm{~S}
\end{array}\right], & \mathrm{w}=\left[\begin{array}{l}
\mathbf{v} \\
\mathbf{\sigma}
\end{array}\right] \\
\mathbf{B}=\left[\begin{array}{cc}
0 & -\mathbf{M} \\
-\mathbf{N} & 0
\end{array}\right], & \mathbf{b}=\left[\begin{array}{l}
\mathbf{b}_{1} \\
\mathbf{b}_{2}
\end{array}\right]
\end{array}
$$

and $I$ is a unit square matrix. It can be checked easily that the governing equations for general plane wave propagation derived in Eqs. (5)-(11) as well as the equations for other plane wave propagation reduced from Eqs. (5)-(11) can be written in the form of Eq. (19). Moreover, A and B are both symmetric in all cases. In particular, $\mathbf{N}=\mathbf{M}^{T}$ and the elements of $\mathbf{N}$ and $\mathbf{M}$ are either one or zero. The same is true for all cylindrical waves. For the most general cylindrical wave propagation, it can be shown, using von Mises' yield condition, that

$$
\begin{array}{ll}
\mathbf{v}=\left[\begin{array}{l}
v_{r} \\
v_{\theta} \\
v_{z}
\end{array}\right], \quad \mathbf{b}_{1}=\left[\begin{array}{c}
\left(\sigma_{1}-\sigma_{2}\right) / r \\
2 \tau_{\theta} / r \\
\tau_{z} / r
\end{array}\right], \quad \mathbf{M}=\left[\begin{array}{lllll}
1 & 0 & 0 & 0 & 0 \\
0 & 0 & 0 & 1 & 0 \\
0 & 0 & 0 & 0 & 1
\end{array}\right], \\
\mathbf{j}=\left[\begin{array}{c}
\sigma_{r} \\
\sigma_{\theta} \\
\sigma_{z} \\
\tau_{\theta} \\
\tau_{z}
\end{array}\right], \quad \mathbf{b}_{2}=\left[\begin{array}{c}
0 \\
v_{r} / r \\
0 \\
-v_{\theta} / r \\
0
\end{array}\right], \quad \mathbf{N}=\mathbf{M}^{T},
\end{array}
$$




$$
\mathrm{S}^{e}=\left[\begin{array}{ccccc}
1 / E & -\nu / E & -\nu / E & 0 & 0 \\
-\nu / E & 1 / E & -\nu / E & 0 & 0 \\
-\nu / E & -\nu / E & 1 / E & 0 & 0 \\
0 & 0 & 0 & 1 / \mu & 0 \\
0 & 0 & 0 & 0 & 1 / \mu
\end{array}\right], \quad \nabla f=\left[\begin{array}{c}
s_{r} \\
s_{\theta} \\
s_{z} \\
2 \tau_{\theta} \\
2 \tau_{z}
\end{array}\right] .
$$

Cristescu [8] has studied an axially symmetric wave propagation in which $v_{r}$ and $v_{\theta}$ are the only nonvanishing velocities. The governing equations can be obtained from Eq. (21) by letting $v_{z} \equiv \tau_{z} \equiv 0$. The particular cases of two-shear cylindrical waves and pressure-shear cylindrical waves have also been derived earlier in [5] but the resulting coefficient matrices were not symmetric. The formulation presented here yields symmetric coefficient matrices for all cylindrical waves.

The analyses in the rest of this paper will be based on the matrix differential equation (19). Although for all plane waves and cylindrical waves the matrices $\mathbf{A}$ and $\mathbf{B}$ are symmetric, the analyses presented in the following do not require the symmetry property of A and B. The yield condition $f$ of Eq. (4) is not restricted to von Mises' yield condition.

3. An identity. In this section, we will derive an identity which is useful in the analyses of the present problem.

Let $\mathbf{P}$ be an $r \times r$ matrix and $\mathbf{g}$ and $\mathbf{h}$ be column vectors with $r$ components. Then, if $a$ is a scalar,

$$
\left\|\mathbf{P}+a \mathbf{h g}^{T}\right\|=\|\mathbf{P}\|+a \mathbf{h}^{T} \mathbf{P}^{*} \mathbf{g}
$$

where $\|\mathbf{P}\|$ is the determinant of $\mathbf{P} . \mathbf{P}^{*}$ is the adjoint matrix of $\mathbf{P}$, i.e. the element $P_{i j}^{*}$ in $\mathrm{P}^{*}$ is the cofactor of the element $P_{i j}$ in $\mathrm{P}$. Hence $\mathrm{P}^{*}$ has the property

$$
\left(\mathbf{P}^{*}\right)^{T} \mathbf{P}=\|\mathbf{P}\| \mathbf{I} .
$$

To prove Eq. (22), we write $\mathbf{P}$ in terms of its columns as

$$
\mathbf{P}=\left[\mathbf{p}_{1}, \mathbf{p}_{2}, \cdots, \mathbf{p}_{r}\right]
$$

where $\mathbf{p}_{1}, \mathbf{p}_{2}, \cdots$ are column vectors. If $g_{1}, g_{2}, \cdots, g_{r}$ denote the components of $\mathbf{g}$, the left-hand side of Eq. (22) can be written as

$$
\left\|\mathbf{P}+a \mathbf{h g}^{T}\right\|=\left\|\mathbf{p}_{1}+a g_{1} \mathbf{h}, \mathbf{p}_{2}+a g_{2} \mathbf{h}, \cdots, \mathbf{p}_{r}+a g_{r} \mathbf{h}\right\| .
$$

Now, by the theory of determinants, it is known that

$$
\begin{gathered}
\left\|\mathbf{p}_{1}+a \mathbf{h}, \mathbf{p}_{2}, \mathbf{p}_{3}, \cdots, \mathbf{p}_{r}\right\|=\|\mathbf{P}\|+a\left\|\mathbf{h}, \mathbf{p}_{2}, \mathbf{p}_{3}, \cdots, \mathbf{p}_{r}\right\|, \\
\left\|\mathbf{h}, a \mathbf{h}, \mathbf{p}_{3}, \mathbf{p}_{4}, \cdots, \mathbf{p}_{r}\right\|=0 .
\end{gathered}
$$

By repeatedly applying Eqs. (26) and (27) to the right-hand side of Eq. (25), we obtain

$$
\begin{aligned}
\left\|\mathbf{P}+a \mathbf{h g}^{\mathrm{T}}\right\|= & \left\|\mathbf{p}_{1}, \mathbf{p}_{2}, \cdots, \mathbf{p}_{r}\right\|+a g_{1}\left\|\mathbf{h}, \mathbf{p}_{2}, \mathbf{p}_{3}, \cdots, \mathbf{p}_{r}\right\| \\
& \quad+a g_{2}\left\|\mathbf{p}_{1}, \mathbf{h}, \mathbf{p}_{3}, \cdots, \mathbf{p}_{r}\right\|+\cdots+a g_{r}\left\|\mathbf{p}_{1}, \mathbf{p}_{2}, \cdots, \mathbf{h}\right\| .
\end{aligned}
$$

With this, it is not difficult to see that Eq. (28) can be written in the form of Eq. (22). This completes the proof. 
For the particular case in which $\mathbf{P}$ is a unit matrix, Eq. (22) reduces to

$$
\left\|\mathbf{I}+a \mathbf{h g}^{T}\right\|=1+a \mathbf{h}^{T} \mathbf{g} .
$$

4. The characteristic equation. The characteristics $c$ of Eq. (19) are the roots of the equation (see [9])

$$
\|c \mathbf{A}-\mathbf{B}\|=0 .
$$

Since

$$
c \mathbf{A}-\mathbf{B}=\left[\begin{array}{cc}
\rho c \mathrm{I} & \mathbf{M} \\
\mathbf{N} & c \mathbf{S}
\end{array}\right]=\left[\begin{array}{cc}
\rho c \mathrm{I} & \mathbf{0} \\
\mathbf{N} & \mathbf{D}
\end{array}\right]\left[\begin{array}{cc}
\mathbf{I} & \mathbf{M} / \rho c \\
\mathbf{0} & \mathbf{I} / \rho c
\end{array}\right],
$$

where

$$
\mathrm{D}=\rho c^{2} \mathrm{~S}-\mathrm{NM},
$$

we have

$$
\|c \mathbf{A}-\mathbf{B}\|=(\rho c)^{n-m}\|\mathbf{D}\| .
$$

Thus, instead of expanding the determinant $\|c \mathbf{A}-\mathbf{B}\|$ which is of order $m+n$, we can expand the determinant $\|\mathbf{D}\|$ which is of order $m$.

If we define

$$
\mathrm{D}^{e}=\rho c^{2} \mathrm{~S}^{e}-\mathrm{NM},
$$

then by Eqs. (16) and (31), we have

$$
\mathbf{D}^{p}=\mathbf{D}^{e}+\rho c^{2} G(k)(\nabla f)(\nabla f)^{T} .
$$

Using the identity derived in the previous section, we obtain

$$
\left\|\mathbf{D}^{p}\right\|=\left\|\mathbf{D}^{e}\right\|+\rho c^{2} G(k)(\nabla f)^{T}\left(\mathbf{D}^{e}\right)^{*}(\nabla f) .
$$

Eq. (35) can be used to study the relative positions of the roots of $\left\|\mathbf{D}^{p}\right\|=0$ and $\left\|\mathbf{D}^{e}\right\|=0$ (see [4], [14]).

5. The elastic-plastic boundary. If $c$ is the speed of an elastic-plastic boundary, then

$$
d \mathbf{w} / d t=\mathbf{w}_{x} c+\mathbf{w}_{\iota}
$$

is the total derivative of $w$ along the boundary. Elimination of $w_{x}$ between Eqs. (19) and (36) yields

$$
(c \mathbf{A}-\mathbf{B}) \mathbf{w}_{\imath}=c \mathbf{b}-\mathbf{B} d \mathbf{w} / d t .
$$

Since $\mathrm{w}$ is continuous across an elastic-plastic boundary, Eq. (37) gives

$$
\left(c \mathbf{A}^{p}-\mathbf{B}\right) \mathbf{w}_{t}^{p}=\left(c \mathbf{A}^{e}-\mathbf{B}\right) \mathbf{w}_{t}^{e}
$$

where the superscripts $e$ and $p$ denote the values in elastic and plastic regions respectively. With Eq. (20), Eq. (38) is equivalent to

$$
\begin{aligned}
& \rho c v_{t}^{p}+M \delta_{t}^{p}=\rho c v_{t}^{e}+M \delta_{t}^{q}, \\
& \mathbf{N v}_{t}^{p}+c \mathbf{S}^{p} \boldsymbol{\delta}_{t}^{p}=\mathbf{N v}_{t}^{e}+c \mathbf{S}^{\bullet} \boldsymbol{\delta}_{t}^{e} \text {. }
\end{aligned}
$$


It is more convenient to write Eqs. (39) and (40) in the following forms, using Eqs. (16) and (18):

$$
\begin{gathered}
\rho c\left(\mathbf{v}_{t}^{e}-\mathrm{v}_{t}^{p}\right)+\mathbf{M}\left(\boldsymbol{\sigma}_{t}^{e}-\boldsymbol{\sigma}_{t}^{p}\right)=0, \\
\mathbf{N}\left(\mathrm{v}_{t}^{e}-\mathrm{v}_{t}^{p}\right)+c \mathbf{S}^{e}\left(\boldsymbol{\sigma}_{t}^{e}-\boldsymbol{\sigma}_{t}^{p}\right)=2 c G(k) k k_{t}^{p}(\nabla f),
\end{gathered}
$$

or

$$
\mathbf{N}\left(\mathbf{v}_{t}^{e}-\mathbf{v}_{\imath}^{p}\right)+c \mathbf{S}^{p}\left(\boldsymbol{\sigma}_{t}^{e}-\boldsymbol{\sigma}_{t}^{p}\right)=2 c G(k) k k_{t}^{e}(\nabla f) .
$$

Eqs. (41) and (42a) yicld

$$
\mathbf{D}^{e}\left(\boldsymbol{\sigma}_{t}^{e}-\boldsymbol{\sigma}_{t}^{p}\right)=2 \rho c^{2} G(k) k k_{t}^{p}(\nabla f)
$$

while Eqs. (41) and (42b) yield

$$
\mathrm{D}^{p}\left(\boldsymbol{\sigma}_{\iota}^{e}-\boldsymbol{\sigma}_{\iota}^{p}\right)=2 \rho c^{2} G(k) k k_{t}^{e}(\nabla f) .
$$

Equation (43a) or (43b) gives a relation between $\boldsymbol{\delta}_{t}^{e}$ and $\boldsymbol{\delta}_{t}^{p}$ on both sides of an elasticplastic boundary. For the case of combined longitudinal and torsional stresses in a tube,

$$
\mathrm{D}^{e}=\left[\begin{array}{cc}
\rho c^{2} / E-1 & 0 \\
0 & \rho c^{2} / \mu-1
\end{array}\right]
$$

and Eq. (43a) reduces to the result obtained in [10].

Equation (43a) can be solved for $\left(\boldsymbol{\delta}_{t}^{e}-\boldsymbol{\delta}_{t}^{p}\right)$ by premultiplying both sides of equation by $\left(\mathrm{D}^{e}\right)^{*^{T}}$ and making use of Eq. (23). Hence

$$
\boldsymbol{\delta}_{t}^{e}-\boldsymbol{\delta}_{t}^{p}=2 \rho c^{2} G(k) k k_{t}^{p}\left(\mathbf{D}^{e}\right)^{* T}(\nabla f) /\left\|\mathbf{D}^{e}\right\| .
$$

Premultiplying once more both sides of Eq. $(44)$ by $(\nabla f)^{T}$ and making use of Eq. (18), we obtain

$$
k_{t}^{e}-k_{\imath}^{p}=\rho c^{2} G(k) k_{\iota}^{p}(\nabla f)^{T}\left(\mathbf{D}^{e}\right)^{* T}(\nabla f) /\left\|D^{e}\right\| .
$$

Since the right-hand side of the equation is a scalar,

$$
(\nabla f)^{T}\left(\mathbf{D}^{e}\right)^{* T}(\nabla f)=(\nabla f)^{T}\left(\mathbf{D}^{e}\right)^{*}(\nabla f),
$$

and by virtue of Eq. (35) we have finally

$$
\frac{k_{t}^{e}}{k_{t}^{p}}=\frac{\left\|\mathrm{D}^{p}\right\|}{\left\|\mathrm{D}^{p}\right\|} .
$$

Eq. (46) gives the ranges of possible speeds for loading and unloading waves provided that $k_{t}^{e}$ and $k_{t}^{p}$ are not both zero. A simplest particular case of Eq. (46) is the case of longitudinal wave propagation in a thin rod studied in [13]. As an illustration, consider the case of combined longitudinal and torsional stresses in a thin-walled tube studied in [10]. Equation (46) becomes, for this case,

$$
\frac{k_{t}^{e}}{k_{t}^{p}}=\frac{\left(c^{2} / c_{f}^{2}-1\right)\left(c^{2} / c_{s}^{2}-1\right)}{\left(c^{2} / c_{0}^{2}-1\right)\left(c^{2} / c_{2}^{2}-1\right)}
$$

where $c_{0}^{2}=E / \rho, c_{2}^{2}=\mu / \rho$, and $c_{f}, c_{s}$ are the roots of $\left\|\mathrm{D}^{p}\right\|=0$. For a loading wave $k_{t}^{e} \geqq 0$ and $k_{t}^{p} \geqq 0$, and the left-hand side of the above equation is nonnegative. In order that 
the right-hand side of the equation remain nonnegative, $c$ can have only one of the following values:

$$
c \leqq c_{s}, \quad c_{2} \leqq c \leqq c_{f}, \quad c_{0} \leqq c,
$$

where use has been made of the fact that $c_{s} \leqq c_{2} \leqq c_{f} \leqq c_{0}$. On the other hand, $k_{t}^{e} \leqq 0$, $k_{t}^{p} \geqq 0$, for an unloading wave, and a similar argument shows that $c$ must satisfy one of the following two conditions:

$$
c_{s} \leqq c \leqq c_{2}, \quad c_{f} \leqq c \leqq c_{0} .
$$

These are the results obtained in [10] and agree with the more general results obtained in [14] for three-dimensional elastic-plastic wave propagation.

The case when both $k_{t}^{e}$ and $k_{t}^{p}$ are zero is discussed next.

First notice that $\boldsymbol{\delta}_{t}^{e}=\boldsymbol{\delta}_{t}^{p}$ implies $k_{t}^{e}=k_{t}^{p}=0$. This follows from Eqs. (41) and (42). The converse, however, is not necessarily true. In other words, $k_{t}^{e}=k_{t}^{p}=0$ does not necessarily imply $\boldsymbol{\delta}_{t}^{e}=\boldsymbol{\sigma}_{t}^{p}$. When $k_{t}^{p}=0$, we have, by Eq. (43a), either $\boldsymbol{\delta}_{t}^{\boldsymbol{e}}=\boldsymbol{\sigma}_{t}^{p}$ or $\left\|\mathbf{D}^{e}\right\|$ $=0$. Similarly, when $k_{t}^{e}=0$, we have, by Eq. (43b), either $\boldsymbol{\delta}_{t}^{e}=\boldsymbol{\delta}_{t}^{p}$ or $\left\|\mathbf{D}^{p}\right\|=0$. Consequently, if $k_{t}^{e}=k_{t}^{p}=0$ we have either $\boldsymbol{\delta}_{t}^{e}=\delta_{t}^{p}$ or $\left\|\mathbf{D}^{p}\right\|=\left\|\mathbf{D}^{e}\right\|=0$. The latter can happen only when the stress state is such that there exists a characteristic which belongs to both elastic and plastic regions and the elastic-plastic boundary is tangential to this common characteristic. For example, in the case of combined longitudinal and torsional waves in a thin-walled tube, $c_{0}$ is a common characteristic for the stress state in which $\sigma=0$ while $\tau$ is arbitrary. Then if $k_{t}^{e}=k_{t}^{p}=0$ but $\boldsymbol{\delta}_{t}^{e} \neq \boldsymbol{\delta}_{t}^{p}, c=c_{0}$.

When $\boldsymbol{\sigma}_{t}^{e}=\boldsymbol{\sigma}_{t}^{p}$, we also have $\mathbf{v}_{t}^{e}=\mathbf{v}_{t}^{p}$ by Eq. (41). Hence $\mathrm{w}_{t}$ is continuous across an elastic-plastic boundary. The total derivative of $\mathrm{w}_{\imath}$ along the boundary is

$$
\frac{d \mathbf{w}_{t}}{d t}=\mathbf{w}_{t x} c+\mathbf{w}_{t t} .
$$

If we differentiate Eq. (19) with respect to $t$, and eliminate $\mathrm{w}_{t x}$ from the above equation, we obtain, after applying the resulting equation to both sides of an elastic-plastic boundary and noticing that $\mathrm{w}_{t}^{e}=\mathrm{w}_{t}^{p}$ :

$$
\left(c \mathrm{~A}^{p}-\mathrm{B}\right) \mathrm{w}_{t t}^{p}+c \mathrm{~A}_{t}^{p} \mathrm{w}_{t}^{p}=\left(c \mathrm{~A}^{e}-\mathrm{B}\right) \mathrm{w}_{\imath t}^{e} .
$$

Since this equation is identical to the equation obtained by differentiating Eq. (38) with respect to $t$, the rest of the analysis can be simplified by differentiating Eqs. (39)-(45) with respect to $t$. In particular, dfferentiation of Eqs. (43) yields, using the fact that $\boldsymbol{\sigma}_{t}^{e}=\boldsymbol{\delta}_{t}^{p}, k_{t}^{e}=k_{t}^{p}=0$,

$$
\begin{aligned}
& \mathbf{D}^{e}\left(\boldsymbol{\sigma}_{t t}^{e}-\boldsymbol{\sigma}_{t t}^{p}\right)=2 \rho c^{2} G(k) k k_{t t}^{p}(\nabla f) \\
& \mathbf{D}^{p}\left(\boldsymbol{\sigma}_{t t}^{e}-\boldsymbol{\sigma}_{t t}^{p}\right)=2 \rho c^{2} G(k) k k_{t \iota}^{e}(\nabla f) .
\end{aligned}
$$

Similarly, if we differentiate both sides of Eq. (45), we obtain, after using Eq. (35),

$$
\frac{k_{t t}^{e}}{k_{t t}^{v}}=\frac{\| \mathbf{D}^{p} !}{\left\|\mathbf{D}^{e}\right\|}
$$

Equations (47) and (48) are identical to Eqs. (43) and (46) respectively with the exception that the order of derivatives is changed. 
From the above derivations we can generalize the results and state them as follows: If $\partial^{n} \boldsymbol{\delta}^{e} / \partial t^{n}=\partial^{n} \boldsymbol{\delta}^{p} / \partial t^{n}$ for $n=1,2, \cdots, \alpha-1$ but $\partial^{\alpha} \boldsymbol{\delta}^{e} / \partial t^{\alpha} \neq \partial^{\alpha} \boldsymbol{\delta}^{p} / \partial t^{\alpha}$, we have

$$
\begin{gathered}
\frac{\partial^{n} k^{e}}{\partial t^{n}}=\frac{\partial^{n} k^{p}}{\partial t^{n}}=0, \quad n=1,2, \cdots, \alpha-1, \\
\mathbf{D}^{e}\left(\frac{\partial^{\alpha} \boldsymbol{\sigma}^{e}}{\partial t^{\alpha}}-\frac{\partial^{\alpha} \boldsymbol{d}^{p}}{\partial t^{\alpha}}\right)=2 \rho c^{2} G(k) k \frac{\partial^{\alpha} k^{p}}{\partial t^{\alpha}}(\nabla f)
\end{gathered}
$$

or

$$
\mathrm{D}^{p}\left(\frac{\partial^{\alpha} \boldsymbol{d}^{\mathcal{e}}}{\partial t^{\alpha}}-\frac{\partial^{\alpha} \boldsymbol{d}^{p}}{\partial t^{\alpha}}\right)=2 \rho c^{2} G(k) k \frac{\partial^{\alpha} k^{e}}{\partial t^{\alpha}}(\nabla f)
$$

and

$$
\frac{\partial^{\alpha} k^{e} / \partial t^{\alpha}}{\partial^{\alpha} k^{p} / \partial t^{\alpha}}=\frac{\left\|\mathbf{D}^{p}\right\|}{\left\|\mathbf{D}^{e}\right\|}
$$

if $\partial^{\alpha} k^{\boldsymbol{e}} / \partial t^{\alpha}$ and $\partial^{\alpha} k^{\mathcal{D}} / \partial t^{\alpha}$ are not both zero. If $\partial^{\alpha} k^{e} / \partial t^{\alpha}=\partial^{\alpha} k^{p} / \partial t^{\alpha}=0$, it is clear from Eqs. (50) and the assumption that $\partial^{\alpha} \partial^{e} / \partial t^{\alpha} \neq \partial^{\alpha} \boldsymbol{\delta}^{p} / \partial t^{\alpha}$,

$$
\left\|\mathbf{D}^{e}\right\|=\left\|\mathbf{D}^{p}\right\|=0 .
$$

For a loading wave,

$$
\frac{\partial^{\alpha} k^{e} / \partial t^{\alpha}}{\partial^{\alpha} k^{p} / \partial t^{\alpha}}\left\{\begin{array}{l}
>0 \text { if } \alpha \text { is an odd integer } \\
<0 \text { if } \alpha \text { is an even integer }
\end{array}\right.
$$

while for an unloading wave,

$$
\frac{\partial^{\alpha} k^{e} / \partial t^{\alpha}}{\partial^{\alpha} k^{p} / \partial t^{\alpha}}\left\{\begin{array}{l}
<0 \text { if } \alpha \text { is an odd integer } \\
>0 \text { if } \alpha \text { is an even integer }
\end{array}\right.
$$

With this, Eq. (51a) furnishes a restriction on the speed of an elastic-plastic boundary as in the particular cases considered in $[10,11,13]$. It should be noticed that Eq. (51a) applies if $\partial^{n} \boldsymbol{\delta} / \partial t^{n}(n=1,2, \cdots, \alpha-1)$ are continuous across an elastic-plastic boundary for a finite segment along the boundary. If $\partial^{n} \delta / \partial t^{n}(n=1,2, \cdots, \alpha-1)$ are continuous across the boundary only at the point concerned but not at other points on the boundary, the left-hand side of Eq. (51a) should be modified. An example of this modification for the particular problem of wave propagation in a rod was given in [12].

6. Discussion. The analyses presented here can be modified to include materials other than isotropic work-hardening. For instance, if the yield function $f$ as expressed by Eq. (4) depends on $\sigma_{i j}$ as well as on $\epsilon_{i j}^{p}$ and other parameters, Eq. (18) should be replaced by

$$
(\nabla f)^{T} \boldsymbol{\sigma}_{t}+\left(\nabla^{\prime} f\right)^{T} \varepsilon_{t}^{p}=2 k k_{t}
$$

where $\nabla f$ and $\nabla^{\prime} f$ are respectively the gradient of $f$ with respect to the components of $\boldsymbol{\delta}$ and $\boldsymbol{\varepsilon}^{p}$. Since $\boldsymbol{\varepsilon}_{t}^{p}=G(k)(\nabla f)(\nabla f)^{T} \boldsymbol{\delta}_{\boldsymbol{t}}$, we have

$$
(\nabla f)^{T} \boldsymbol{\sigma}_{t}=2 k k_{t} / H
$$

where $H=1+G(k)\left(\nabla^{\prime} f\right)^{T}(\nabla f)$. With Eq. (18) replaced by Eq. (54), the rest of the analyses remain essentially the same with minor modifications whenever necessary. In particular, the results obtained in Eqs. (35), (46), (48), (51) and (52) remain unchanged. 
Acknowledgments. The work presented here was done at Stanford University during the author's visit in 1967-68 and was supported by the Ballistic Research Laboratories, Aberdeen Proving Ground, Maryland, through Contract DA-04-200-AMC-659(X) with Stanford University. The author is greatly indebted to Professor E. H. Lee for making the visit possible and for valuable discussions on many subjects, including the present work, during his stay at Stanford University.

\section{References}

1. R. Hill, The mathematical theory of plasticity, Clarendon Press, Oxford, 1950

2. H. H. Bleich and I. Nelson, Plane waves in an elastic-plastic half-space due to combined surface pressure and shear, J. Appl. Mech. 33, 149-158 (1966)

3. Ning Nan, Elastic-plastic waves for combined stresses, Tech. Rep. No. 184, Dept. of Appl. Mech., Stanford University, Stanford, Calif., July 1968

4. T. C. T. Ting and Ning Nan, Plane waves due to combined compressive and shear stresses in a halfspace, J. Appl. Mech. 36, 189-197 (1969)

5. Jeffrey T. Fong, Elastic-plastic wave in a half-space of a linearly work-hardening material for coupled shear loadings, Tech. Rept. No. 161, Div. of Eng. Mech., Stanford University, Stanford, Calif., May 1966

6. T. C. T. Ting, Interaction of shock waves due to combined two shear-loadings, Int. J. Solids Structures 5, 415-435 (1969)

7. R. J. Clifton, An analysis of combined longitudinal and torsional plastic waves in a thin-walled tube, Fif th U. S. National Congress of Appl. Mech., June 1966, pp. 465-480

8. N. Cristescu, Some observations about the case of plane axial-symmetric deformations in the dynamic problem of plasticity (Prandtl-Reuss theory), Com. Acad. R. P. Romane (1) 6, 19-28 (1956)

9. R. Courant and D. Hilbert, Methods of mathematical physics, Vol. II, Interscience, New York, 1962

10. R. J. Clifton, Elastic-plastic boundaries in combined longitudinal and torsional plastic wave propagation, J. Appl. Mech. 35, 782-786 (1968)

11. R. J. Clifton and T. C. T. Ting, The elastic-plastic boundary in one-dimensional wave propagation, J. Appl. Mech. 35, 812-814 (1968)

12. T. C. T. Ting, On the initial slope of elastic-plastic boundaries in longitudinal wave propagation in a rod, Tech. Rept. No. 187, Dept. of Appl. Mech., Stanford University, Stanford, Calif., Sept. 1968

13. E. H. Lee, $A$ boundary value problem in the theory of plastic wave propagation, Quart. Appl. Math. 10, 335-346 (1953)

14. Jean Mandel, Ondes plastiques dans un milieu indéfini à trois dimensions, J. Mécanique (1) 1, 3-30 (1962) 